\title{
Dependence of Reflection Wavelength Shift of Resin-embedded Grating Fiber on Resin Material
}

\author{
Hironori Kumazaki, ${ }^{1 *}$ Takato Sugiyama, ${ }^{2}$ and Hisakazu Oguri ${ }^{3}$ \\ ${ }^{1}$ Department of Electrical and Computer Engineering, National Institute of Technology, Gifu College, \\ 2236-2 Kamimakuwa, Motosu, Gifu 501-0495, Japan \\ ${ }^{2}$ Kawaju Gifu Engineering Co., Ltd. 2 Kawasaki, Kagamihara, Gifu 504-0971, Japan \\ ${ }^{3}$ Department of Mechanical Engineering, National Institute of Technology, Gifu College, \\ 2236-2 Kamimakuwa, Motosu, Gifu 501-0495, Japan
}

(Received May 25, 2017; accepted August 29, 2017)

Keywords: fiber Bragg grating, reactive ion etching, bending deflection sensor

The dependence of the center reflection wavelength shift of a resin-embedded fiber Bragg grating (FBG) on the resin material was investigated for the use of the grating fiber as a bending deflection sensor $\left(4 \times 8 \times 40 \mathrm{~mm}^{3}\right)$. The reflection center wavelength for an FBG embedded in acrylic adhesive shifted linearly by up to $3.1 \mathrm{~nm}$ owing to the deflection caused by a bend feeder displacement of $1.2 \mathrm{~mm}$. These wavelength shifts were approximately 60 times larger than those for an etched FBG with one side of the cladding etched to within $11 \mu \mathrm{m}$ of the core and 33 times larger than those for an FBG embedded in a modified silicon adhesive. These results depend primarily on Young's modulus of the resin material used to embed the FBG.

\section{Introduction}

Curvature measurements are important in various applications, ranging from the structural monitoring to the physical analysis of buildings or mechanical systems. Optical fiber sensors, which have advantages over conventional sensors, such as electrically passive operation, magnetic interference (MI) immunity, high sensitivity, and low cost, have been employed for this purpose. In particular, fiber gratings have been widely investigated for use in curvature measurements. Long-period gratings (LPGs) are highly sensitive to curvature-induced wavelength shifts and changes in the amplitude of the cladding mode resonances. However, they also exhibit high-temperature cross-sensitivity and a wide bandwidth of the cladding mode resonances, which causes measurement errors and difficulty in multiplexing. ${ }^{(1,2)}$ On the other hand, chirped fiber Bragg gratings (CFBGs) or linearly etched fiber Bragg gratings (FBGs) can be used for curvature measurements because the power reflectivity changes upon bending. Substantial flexible beams for embedded CFBGs or etched FBGs are essential for this type of sensor. $^{(3,4)}$ A curvature sensor based on an FBG with an asymmetrical cross section was proposed and fabricated by anisotropic etching. ${ }^{(5)}$ The application of a bending stress to this fiber is equivalent to inducing a longitudinal stress in the fiber core, which alters the grating

*Corresponding author: e-mail: kumazaki@gifu-nct.ac.jp

http://dx.doi.org/10.18494/SAM.2018.1640 
period and effective refractive index. This method has potential for the measurement of the degree of curvature of an independent optical fiber without using other structures. However, there are still issues that must be overcome prior to their application, such as measurement sensitivity and durability, in various environments.

In this study, shifts in center reflection wavelength induced by bending deflection were compared for an etched FBG and an FBG embedded in resin. Furthermore, adhesive resins with different Young's moduli were evaluated as constituent materials for deflection sensors based on FBGs embedded in resin.

\section{Sensor Configuration and Experimental Setup}

Figure 1 shows the structure of the embedded sensor. The FBG with a length of $10 \mathrm{~mm}$ is embedded along the long side into the middle of a 55-mm-long resin triangular prism, whose cross section is a right-angled isosceles triangle with a height of $4 \mathrm{~mm}$. Three different adhesive materials were used to construct three embedded sensors with the same configuration and size, namely, acrylic adhesive, epoxy adhesive, and modified silicon polymer adhesive, and the sensors were denoted Embed-A, Embed-E, and Embed-S, respectively. Figures 2 and 3 show the structure of the etched sensor (denoted Etched) and the sensor cross section, respectively. For the etched sensor, one side of the fiber was etched by reactive ion etching (RIE) along a length of more than $15 \mathrm{~mm}$, which contained a grating length of $10 \mathrm{~mm}$. RIE was performed at

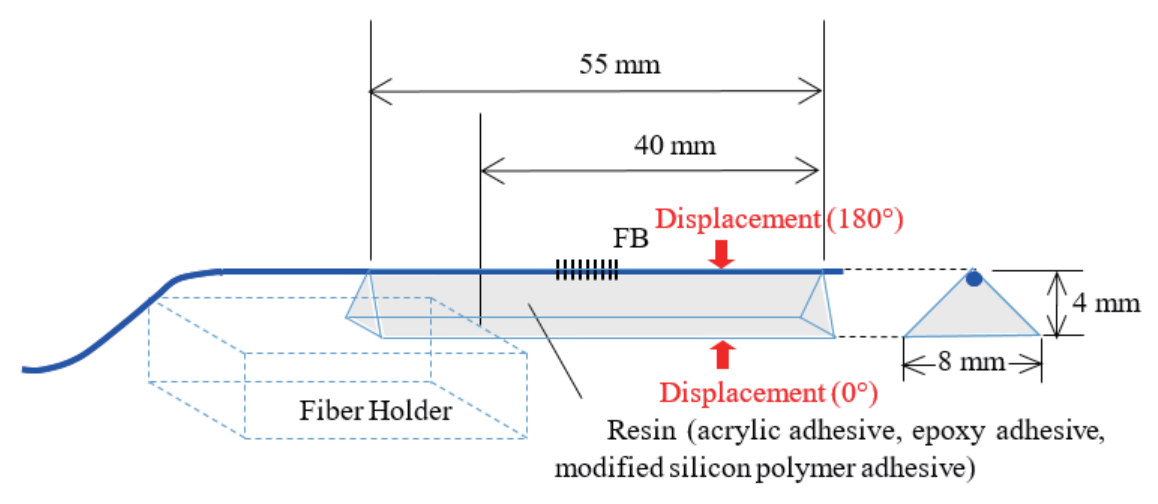

Fig. 1. (Color online) Structure of the embedded sensor.

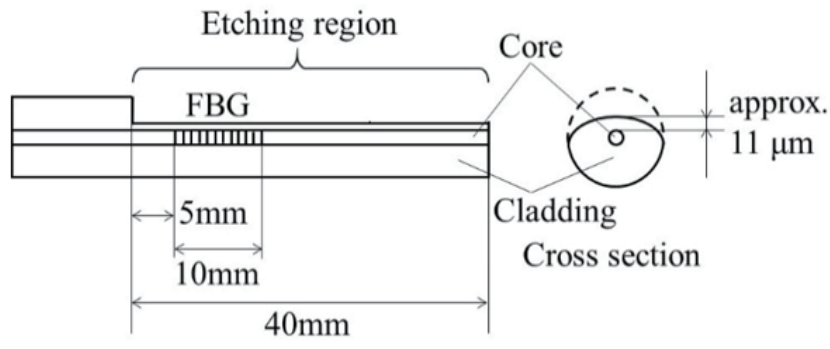

Fig. 2. Structure of the etched sensor.

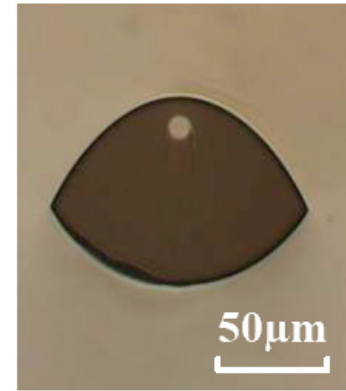

Fig. 3. (Color online) Cross section of the etched sensor. 
a pressure of $13 \mathrm{~Pa}$ and an RF power of $150 \mathrm{~W}$. One side of the cladding was etched to within 11 $\mu \mathrm{m}$ of the core. FBGs were fabricated in hydrogen-loaded single-mode fibers using a scanning phase-mask technique with a $\mathrm{KrF}$ excimer laser with an output wavelength of $248 \mathrm{~nm}$.

The center reflection wavelengths were 1545.8, 1548.4, 1550.3, and 1549.3 nm for Embed-A, Embed-E, Embed-S, and Etched, respectively. Figure 4 shows the experimental setup used for the deflection sensor based on a 40-mm-long cantilever with the FBG embedded in resin. Broadband light from an amplified spontaneous emission (ASE) light source was incident on the input port and passed through an optical circulator before reaching the grating fiber. Light at the Bragg wavelength was reflected and extracted at the optical circulator. The reflected light spectra were measured for two bending deflections, as described below, on a flat in-plane bend using an optical spectrum analyzer. After controlling the bend direction using a rotation stage, the FBG sensor was curved downward by displacing the bend feeder that applied force to one point on the sensor $5 \mathrm{~mm}$ away from the tip of the sensor. The bend direction was defined relative to the original orientation of $0^{\circ}$ where the maximum compressive distortion occurred in the core with the same bend, that is, when the fiber was bent in the same direction as the etched surface for the etched sensor and when the sensor was bent vertically upward for the embedded sensor, as shown in Fig. 1. The shifts in the center reflection wavelength $t$ for Embed-A, Embed-E, Embed-S, and Etched were measured as a function of the displacement of the bend feeder in the 0 and $180^{\circ}$ directions. All hardening treatments for resin and all experiments were carried out under the same atmosphere at $25^{\circ} \mathrm{C}$.

\section{Theoretical Calculation Procedures}

The center reflection wavelength shift caused by the bending of the embedded FBG sensor is now discussed theoretically. The theoretical center wavelength shift $\Delta \lambda$ for the applied bending strain $\varepsilon$ is given as ${ }^{(6)}$

$$
\Delta \lambda=\lambda_{s}\left(1-p_{e}\right) \varepsilon
$$

where $\lambda_{s}$ is the center reflection wavelength of the straight grating fiber and $p_{e}$ is the

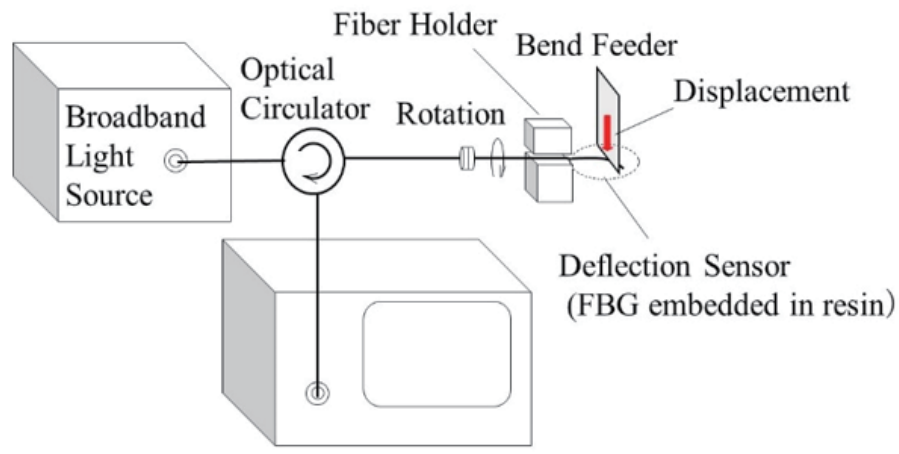

Optical Spectrum Analyzer

Fig. 4. (Color online) Experimental setup used for deflection. 
photoelastic coefficient of the fiber. For a silica core fiber, the value of $\left(1-p_{e}\right)$ is usually $0.78 .^{(6)}$ The bending strain $\varepsilon$ could be calculated theoretically using

$$
\varepsilon= \pm \frac{y_{c}}{R}
$$

where $\pm R$ is the radius of curvature for tensile or compressive stress and $y_{\mathrm{c}}$ is the distance between the center of the core and the centroid of the sensor cross section, as shown in Fig. 5, where a schematic of the cross-sectional model for an embedded FBG sensor is presented. This type of sensor could be considered as a composite beam structure composed of an optical fiber and the embedding resin. The centroid of the sensor cross section and $y_{c}(=Y-\bar{y})$ were first calculated theoretically using Eq. (3) under the assumption that no slip occurred between the optical fiber and resin. The distance between the bottom face of the resin and the centroid of the sensor cross section, $\bar{y}$ in Fig. 5, is expressed as

$$
\bar{y}=\frac{\sum E_{i} \int_{A_{i}} Y d A}{\sum E_{i} A_{i}}
$$

where $d A$ is a small cross-sectional area at an arbitrary distance from the bottom face of the resin, and $E_{i}$ and $A_{i}$ are Young's modulus and the cross-sectional area, respectively. The subscript $i$ is an index for the various sensor materials, such as the optical fiber $(i=1)$ and resin $(i=2)$. Table 1 shows the main characteristics of their adhesive materials, calculated results for $y_{c}$ and the center reflection wavelength for that the FBG embedded in a triangular prism resin

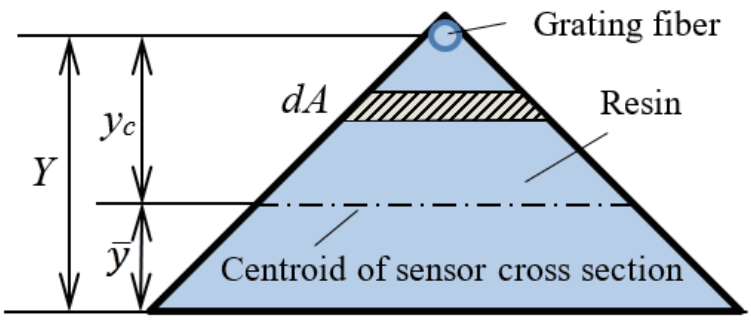

Fig. 5. (Color online) Composite beam model of the sensor cross section for calculating theoretical values.

Table 1

Main characteristics of adhesive materials, calculated results for $y_{\mathrm{c}}$ and center reflection wavelength of the FBG.

\begin{tabular}{lcccccc}
\hline $\begin{array}{l}\text { Type of } \\
\text { adhesive material }\end{array}$ & $\begin{array}{c}\text { Young's } \\
\text { modulus } \\
(\mathrm{Pa})\end{array}$ & $\begin{array}{c}\text { Tensile shear } \\
\text { adhesive strength } \\
\left(\mathrm{N} / \mathrm{mm}^{2}\right)\end{array}$ & $\begin{array}{c}\text { T-type peeling } \\
\text { adhesive strength } \\
(\mathrm{N} / \mathrm{mm})\end{array}$ & $\begin{array}{c}\text { Linear } \\
\text { expansion } \\
\text { coefficient }\end{array}$ & $\begin{array}{c}y_{c} \\
(\mu \mathrm{m})\end{array}$ & $\begin{array}{c}\text { Center reflection } \\
\text { wavelength of } \\
\text { the FBG }(\mathrm{nm})\end{array}$ \\
\hline $\begin{array}{c}\text { Modified silicon } \\
\text { polymer adhesive } \\
\text { (Cemedine Super X) }\end{array}$ & $1.4 \times 10^{6}$ & 3.5 & 3.4 & $2.1 \times 10^{-4}$ & 60 & 1550.3 \\
$\begin{array}{c}\text { Epoxy adhesive } \\
\text { (Cemedine EP330) }\end{array}$ & $4.0 \times 10^{7}$ & 17.5 & 0.47 & $6.7 \times 10^{-5}$ & 240 & 1548.4 \\
$\begin{array}{c}\text { Acrylic adhesive } \\
(\text { Cemedine Y630D) }\end{array}$ & $2.4 \times 10^{9}$ & 24.7 & 4.6 & $1.2 \times 10^{-4}$ & 2550 & 1545.8 \\
\hline
\end{tabular}


having an isosceles right angle base with a height of $4 \mathrm{~mm}$. The theoretical results for $y_{c}$ were mainly affected by Young's modulus for resin, $E_{2}$, of $E_{1}\left(7.3 \times 10^{10} \mathrm{~Pa}\right)$ for the quartz optical fiber.

Next, we describe the procedure used to convert the bending deflection to the radius of curvature for the cantilever-embedded FBG using the bend model shown in Fig. 6. It was supposed that a load $P$ was applied to the cantilever by the bend feeder at a point located at a distance $l$ from the edge of the fiber holder, with $x$ being the distance from the edge of the fiber holder to the center of the FBG. The load $P$ is expressed as a function of the deflection $\delta$ as

$$
P=\frac{3 E I_{z}}{l^{3}} \delta
$$

where $E, I_{z}$, and $l$ are Young's modulus, the geometrical moment of inertia, and the distance between the supporting point and the point of applied load, respectively. When the function that represents the deflection curve for the cantilever-embedded FBG is defined as $y$, the radius of curvature $R$ is expressed as

$$
R=\frac{\left(1+y^{\prime}\right)^{\frac{3}{2}}}{y^{\prime \prime}}
$$

where $y^{\prime}$ and $y^{\prime \prime}$ are calculated from the boundary conditions using

$$
\begin{gathered}
y^{\prime}=\frac{3\left(x^{2}-l^{2}\right)}{2 l^{3}} \delta, \\
y^{\prime \prime}=\frac{3 x}{l^{3}} \delta .
\end{gathered}
$$

The radius of curvature $R$ was calculated as a function of $x$ and $\delta$ by substituting Eqs. (4), (6), and (7) into Eq. (5). Finally, the theoretical center wavelength shift $\Delta \lambda$ was calculated using Eq. (1) after the calculation of the bending strain $\varepsilon$ using Eq. (2).

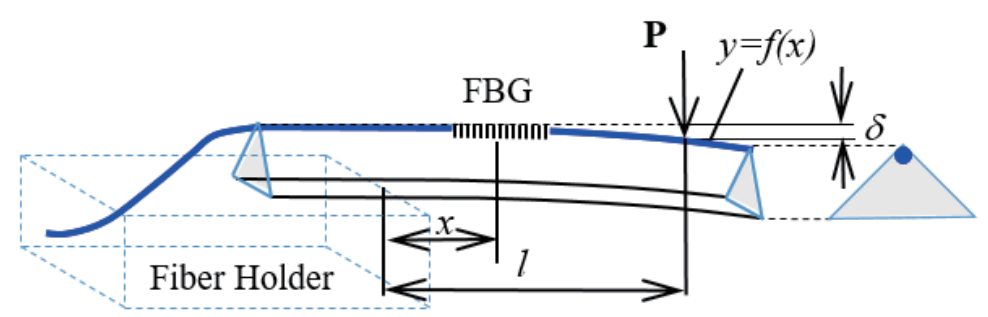

Fig. 6. (Color online) Approximation model to convert bending deflection to radius of curvature for the cantilever-embedded FBG. 


\section{Experimental Results and Discussion}

Figure 7 shows the center reflection wavelength shifts for Embed-A, Embed-E, Embed-S, and Etched as a function of the bend feeder displacement in the 0 and $180^{\circ}$ bend directions. Each wavelength shift increased linearly with the bend feeder displacement. The shifts for bend feeder displacement of $1.2 \mathrm{~mm}$ for Embed-A, Embed-E, Embed-S, and Etched were 3.1, 0.39, 0.10, and $0.05 \mathrm{~nm}$, respectively. The rate of the center reflection wavelength shift for the bend feeder displacement was approximately $2.6 \mathrm{~nm} / \mathrm{mm}$ for Embed-A, $3.3 \times 10^{-1} \mathrm{~nm} / \mathrm{mm}$ for Embed-E, 8.0 $\times 10^{-2} \mathrm{~nm} / \mathrm{mm}$ for Embed-S, and $4.2 \times 10^{-2} \mathrm{~nm} / \mathrm{mm}$ for Etched. The shift rate for Embed-A was approximately 60 times larger than that for Etched and 33 times larger than that for Embed-S for any deflection examined in this study. Since the wavelength in a stationary state can be measured with a resolution of $0.01 \mathrm{~nm}$, Embed-A can be used for deflection measurement with a resolution of about $4 \mu \mathrm{m}$. Figure 8 shows the measurement resolution as a function of the ratio of Young's moduli for resin and optical fiber, $E_{2} / E_{1}$. The measurement resolution with the unit of $\mu \mathrm{m}$ is approximated as $0.87822 \times\left(E_{2} / E_{1}\right)^{\wedge}(-0.45882)$ from the approximate expression based on the acquired measurement data shown by the symbols in Fig. 8. A larger ratio $\left(E_{2} / E_{1}\right)$ should result in larger $y_{c}$, which is necessary to improve the measurement resolution for bending deflection on an embedded FBG sensor. A temperature change of $1{ }^{\circ} \mathrm{C}$ also causes the same center reflection wavelength shift as a bend feeder displacement of about $4 \mu \mathrm{m}$ on Embed-A.

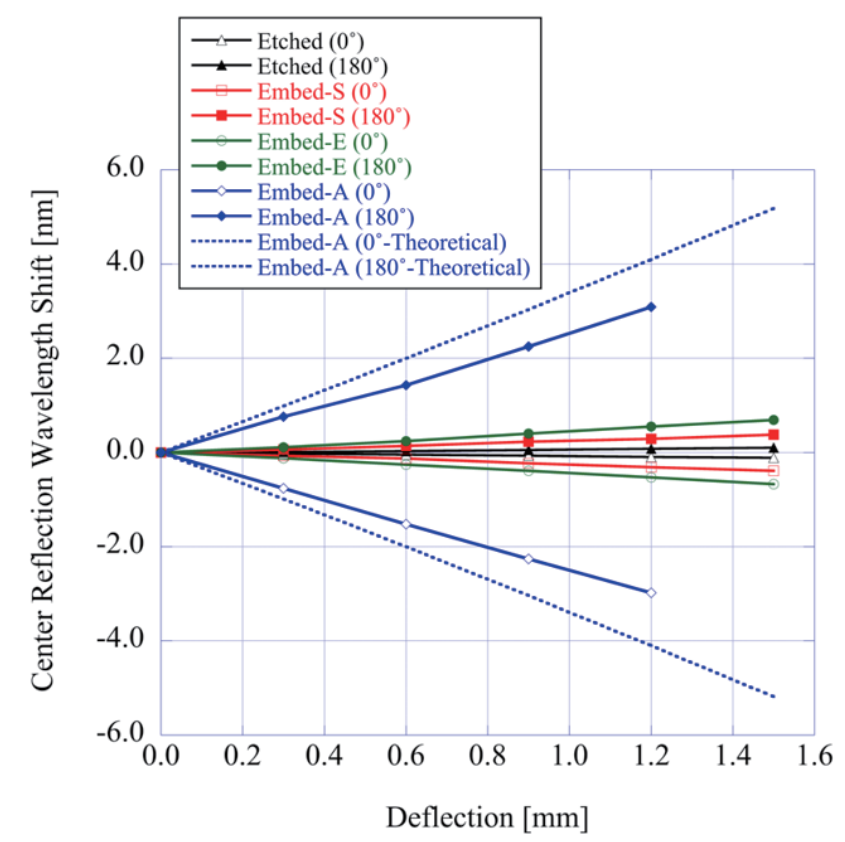

Fig. 7. (Color online) Center reflection wavelength shift as a function of bend feeder displacement. 


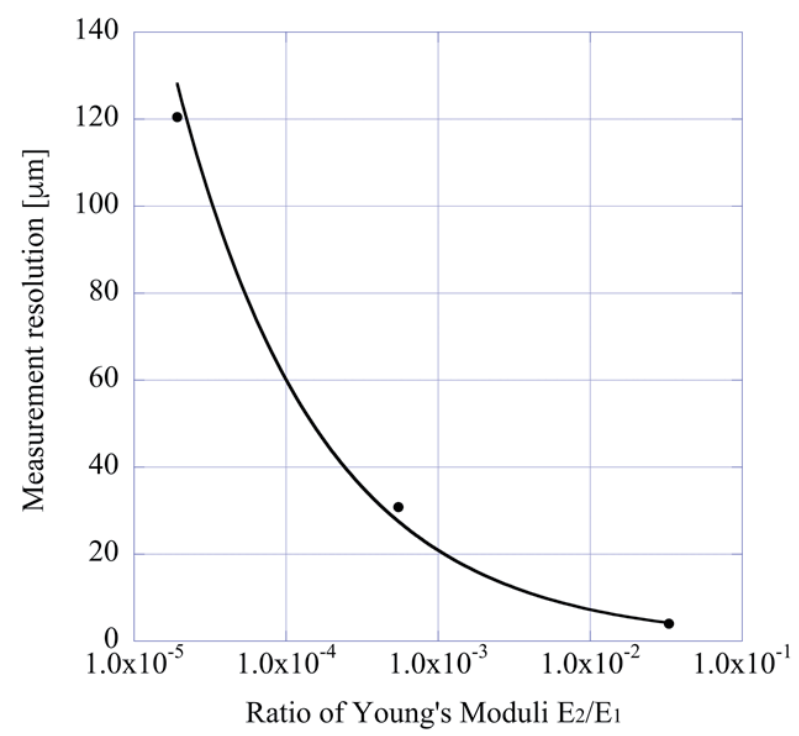

Fig. 8. Measurement resolution as a function of ratio, $E_{2} / E_{1}$, of Young's moduli for resin and optical fiber.

The theoretical results for center reflection wavelength shifts were obtained by calculating the deformation of the core from the cross section of the FBG-embedded resin or the etched FBG, assuming the gauge factor $G$ to be 0.78 . The theoretical shift rate was approximately $3.6 \mathrm{~nm} / \mathrm{mm}$ for Embed-A, $3.4 \times 10^{-1} \mathrm{~nm} / \mathrm{mm}$ for Embed-E, $8.4 \times 10^{-2} \mathrm{~nm} / \mathrm{mm}$ for Embed-S, and $4.1 \times 10^{-2} \mathrm{~nm} / \mathrm{mm}$ for Etched. The experimental shift in the center reflection wavelength is very close to the theoretically predicted shift. The differences were within 5\% for Embed-E, Embed-S, and Etched. Therefore, it can be said that this model and calculation method for theoretical results are appropriate for evaluating these sensors. However, the experimental shifts were $28 \%$ smaller than the theoretical shift, which is shown as the dotted line in Fig. 7 for the Embed-A sensor. This discrepancy may be due to an inadequate deflection induced by the grating because the hardened acrylic adhesive was very stiff and a slight slip could be induced at the fixed portion of the sensor cantilever on the fiber holder.

According to both the experimental and theoretical results, larger wavelength shifts were obtained using bending deflection sensors of the same shape for the FBG embedded in resin when Young's modulus of the resin material used to embed the FBG was higher. However, if the stiffness of the resin is very high, then the sensor will snap off or be damaged by the deflection.

\section{Conclusions}

FBGs embedded in various resin materials were examined as optical bend sensors. Each wavelength shift increased linearly with the bending deflection and was almost in agreement with the theoretical values calculated using a composite beam model. Embed-A with the size of $4 \times 8 \times 40 \mathrm{~mm}^{3}$ can be used for deflection measurement with a resolution of about $4 \mu \mathrm{m}$. The wavelength shifts obtained for bending deflection sensors based on the FBG embedded in resin increased with Young's modulus of the resin material, unless the sensor snapped off. 


\section{Acknowledgments}

This work was supported by Suzuki Foundation in Japan.

\section{References}

1 T. Allsop, K. Kalli, K. Zhou, Y. Lai, G. Smith, M. Dubov, D. J. Webb, and I. Bennion: Opt. Commun. 281 (2008) 5092.

2 L. Y. Shao, J. Zhao, X. Dong, H. Y. Tam, C. Lu, and S. He: Appl. Opt. 47 (2008) 1549.

3 W. Zhou, X. Dong, K. Ni, C. C. Chan, and P. Shum: Sens. Actuators, A 157 (2010) 15.

4 Y. S. Yu, Z. Y. Zhao, Z. C. Zhuo, W. Zheng, Y. Qian, and Y. S. Zhang: Microwave Opt. Technol. 43 (2004) 414.

5 H. Kumazaki, M. Hiramatsu, H. Oguri, S. Inaba, and K. Hane: IEEJ Trans. Sens. Micromach. 134 (2014) 41.

6 Q. Wang, G. Farrell, T. Freir, G. Rajan, and P. Wang: Opt. Lett. 31 (2006) 1785.

\section{About the Authors}

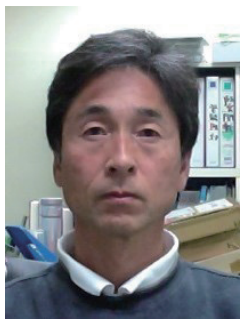

Hironori Kumazaki received his M.S degree from Nagaoka University of Technology in 1983 and Dr. Engineering degree from Tohoku University in 2002. From 1983 to 1994, he worked at Nissin Electric Co., Ltd. Since 1994, he has been working at the Department of Electrical and Computer Engineering in Gifu National College of Technology, and is currently engaged in the research and development of optical microsensors. Since 2004, he has been a professor in the Department of Electrical and Computer Engineering in Gifu National College of Technology.

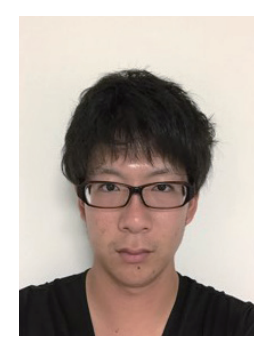

Takato Sugiyama graduated from the Gifu National College of Technology Advanced Course "Course of Electronic System Engineering" and received his B.E degree from National Institution of Academic Degrees University Evaluation in 2015. Since 2015, he has been working in the Electronic \& Information Engineering Department of the Aircraft Engineering Division at Kawaju Gifu Engineering Co., Ltd., and is currently engaged in the design and production of aerospace equipment and systems.

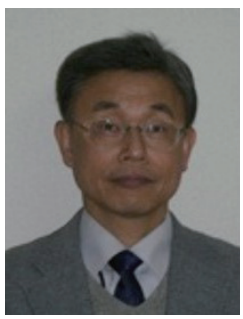

Hisakazu Oguri received his B.E degree and M.S degree from Toyohashi University of Technology in 1982 and 1984, respectively. Since 1984, he has been working at the Department of Mechanical Engineering in Gifu National College of Technology, and is currently engaged in the research of the strength of materials and the development of a measurement method of the strength of materials. Since 2008, he has been a professor in the Department of Mechanical Engineering in Gifu National College of Technology. 